\title{
ENVELOPPES CONVEXES DES PROCESSUS GAUSSIENS
}

\author{
Youri DAVYDOV \\ Laboratoire de statistique et probabilités, bât. M2, FRE-CNRS 2222, \\ Université des sciences et technologies de Lille, 59655 Villeneuve d'Ascq cedex, France
}

Reçu le 3 mars 2001, revisé le 14 janvier 2002

RÉSUMÉ. - On étudie la structure de l'ensemble ext $\left(V_{X}\right)$ des points extrémaux de l'enveloppe convexe $V_{X}$ de la trajectoire d'un processus $X$ :

$$
V_{X}=\operatorname{Conv}\{(t, x) \mid t \in[0,1], x=X(t)\}
$$

Pour $X$ gaussien centré à accroissements stationnaires on montre que :

- L'ensemble ext $\left(V_{X}\right)$ est négligeable, si $X$ est non dérivable.

- Si $X$ est un processus absolument continu, $X(t)=X(s)+\int_{s}^{t} X^{\prime}(u) d u$, et sa dérivée $X^{\prime}$ est continue mais non dérivable, alors l'ensemble $\operatorname{ext}\left(V_{X}\right)$ est aussi négligeable, mais de plus, il est cantorien.

On montre aussi que les propriétés ci-dessus sont stables par transformations du type $Y(t)=$ $f(X(t))$, si $f$ est une fonction suffisamment lisse.

() 2002 Éditions scientifiques et médicales Elsevier SAS

Mots Clés : Processus gaussiens; Enveloppes convexes; Ensemble de Cantor

ABStRACT. - Let $X=\{X(t), t \in[0,1]\}$ be a process on $[0,1]$ and

$$
V_{X}=\operatorname{Conv}\{(t, x) \mid t \in[0,1], x=X(t)\}
$$

be the convex hull of its path.

The structure of the set $\operatorname{ext}\left(V_{X}\right)$ of extreme points of $V_{X}$ is studied. For a Gaussian process $X$ with stationary increments it is proved that:

- The set $\operatorname{ext}\left(V_{X}\right)$ is negligible if $X$ is non-differentiable.

- If $X$ is absolutely continuous process and its derivative $X^{\prime}$ is continuous but nondifferentiable, then $\operatorname{ext}\left(V_{X}\right)$ is also negligible and moreover it is a Cantor set.

It is proved also that these properties are stable under the transformations of the type $Y(t)=$ $f(X(t))$, if $f$ is a sufficiently smooth function.

() 2002 Éditions scientifiques et médicales Elsevier SAS

MSC: 60G15; 60G17; 60D05

Keywords: Gaussian processes; Convex hulls; Cantor sets 


\section{Introduction}

Soit $X=\{X(t), t \in[0,1]\}$ un processus réel mesurable sur $[0,1]$. On va étudier l'ensemble $\operatorname{ext}\left(V_{X}\right)$ des points extrémaux de l'enveloppe convexe $V_{X}$ de la trajectoire de $X$ :

$$
V_{X}=\operatorname{Conv}\{(t, x) \mid t \in[0,1], x=X(t)\} .
$$

L'intérêt de l'étude des enveloppes convexes repose sur des motivations multiples. Avant tout, une telle étude ferait partie de la recherche des probabilités naturelles dans l'espace des sous-ensembles compacts convexes de $\mathbb{R}^{d}$, concentrées sur des familles d' ensembles dont la frontière possède des propriétés non triviales. Ce programme a été partiellement réalisé dans [6] et développé dans [5] où on étudiait les lois limites des sommes de Minkowski des segments aléatoires.

Une autre motivation, encore plus importante, c'est qu'on a besoin des enveloppes convexes pour construire des solutions de l'équation de Burgers avec conditions initiales aléatoires. Les points extrémaux dans ce cas s'interprètent comme les points de chocs des particules (voir $[1,11,12]$ et leurs références).

Les premiers articles consacrés aux enveloppes convexes étaient [10] et [8] où Pitman et Groenboom ont démontré pour le mouvement brownien standard $(X=W)$ un résultat remarquable : avec probabilité 1 l'ensemble ext $\left(V_{X}\right)$ est dénombrable n'ayant que deux points d'accumulation 0 et 1 .

Ensuite Sinai [13] a montré que pour le mouvement brownien intégré $X(t)=$ $\int_{0}^{t} W(s) d s$ l'ensemble $\operatorname{ext}\left(V_{X}\right)$ est un ensemble de mesure de Lebesgue zéro, mais cantorien, c.à.d. fermé, nulle part dense et sans points isolés (donc, de puissance de continu). De plus, il a démontré que la dimension de Hausdorff de cet ensemble est égale à $1 / 2$ p.s.

Le résultat fin de Bertoin [2], traduit dans le langage des enveloppes convexes, signifie que pour les processus $\alpha$-stables avec $\alpha>1 / 2$, la structure de l'ensemble $\operatorname{ext}\left(V_{X}\right)$ est discrète p.s.

Le but de cet article est d'étudier les enveloppes convexes des processus gaussiens et de montrer que la négligeabilité de l'ensemble $\operatorname{ext}\left(V_{X}\right)$ est un phénomène typique. Les résultats essentiels sont les suivants. Pour un processus $X$ gaussien centré, à accroissements stationnaires, on montre que :

- L'ensemble ext $\left(V_{X}\right)$ est p.s. négligeable, si $X$ est non dérivable.

- Si $X$ est un processus absolument continu, $X(t)=X(s)+\int_{s}^{t} X^{\prime}(u) d u$, et sa dérivée $X^{\prime}$ est non dérivable, alors l'ensemble $\operatorname{ext}\left(V_{X}\right)$ est aussi p.s. négligeable, mais de plus, il est cantorien.

- Les propriétés ci-dessus sont stables par transformations du type $Y(t)=f(X(t))$, si $f$ est une fonction suffisamment lisse.

Pour être plus précis, dans toute la suite on utilisera la terminologie suivante. On dira qu'un processus $X$ est :

- continu, si p.s. ses trajectoires sont continues sur $[0,1]$;

- dérivable, si p.s. ses trajectoires sont dérivables en tout point de [0,1];

- non dérivable, si p.s. la dérivée $X^{\prime}(t)$ n'existe pas presque partout dans $[0,1]$.

Dans la section 2, on propose des conditions simples suffisantes pour que $\operatorname{ext}\left(V_{X}\right)$ soit négligeable. La section 3 est consacré aux processus gaussiens. Les enveloppes convexes 
des processus gaussiens transformés sont étudiées dans la section 4 . La cinquième et dernière section est réservée pour une discussion.

\section{Enveloppes convexes des fonctions et processus}

\subsection{Fonctions}

Soit $f:[0,1] \rightarrow \mathbb{R}^{1}$ une fonction. On note $\Gamma_{f}$ son graphe, $V_{f}$ l'enveloppe convexe du $\Gamma_{f}$ et $\operatorname{ext}\left(V_{f}\right)$ l'ensemble des points extrémaux de $V_{f}$.

On s'intéresse à la structure de l'ensemble $E_{f}=\pi$ (ext $V_{f}$ ), où $\pi$ est la projection canonique de $[0,1] \times \mathbb{R}^{1}$ dans $[0,1], \pi(x, y)=x$, et à l'estimation de la mesure de Lebesgue $\lambda$ de $E_{f}$.

Notre approche est basée sur la remarque suivante.

Lemme 1. - Soit $t \in(0,1)$. Supposons qu'il existe des points $s_{1}, s_{2}, 0 \leqslant s_{1}<t<$ $s_{2} \leqslant 1$ et $u_{1}, u_{2}, 0 \leqslant u_{1}<t<u_{2} \leqslant 1$, tels que

$$
\frac{f\left(s_{1}\right)-f(t)}{s_{1}-t}<\frac{f\left(s_{2}\right)-f(t)}{s_{2}-t}
$$

et

$$
\frac{f\left(u_{1}\right)-f(t)}{u_{1}-t}>\frac{f\left(u_{2}\right)-f(t)}{u_{2}-t} .
$$

Alors le point $(t, f(t))$ appartient à $V_{f}^{o}$, l'intérieur de $V_{f}$.

Démonstration. - Il suffit de remarquer que le segment joignant les points $\left(s_{1}, f\left(s_{1}\right)\right)$ et $\left(s_{2}, f\left(s_{2}\right)\right)$, se trouve au-dessus du point $(t, f(t))$, tandis que le segment joignant $\left(u_{1}, f\left(u_{1}\right)\right)$ et $\left(u_{2}, f\left(u_{2}\right)\right)$ passe au-dessous.

Proposition 1. - Supposons que la fonction $f$ soit continue et que la condition suivante soit vérifiée :

(A) Pour presque tout $t \in[0,1]$, il existe $\varepsilon_{t}>0$ tel que $\forall 0<\varepsilon<\varepsilon_{t}$

$$
\inf _{s \in(t, t+\varepsilon)} f(s)<f(t)<\sup _{s \in(t, t+\varepsilon)} f(s)
$$

et

$$
\inf _{s \in(t-\varepsilon, t)} f(s)<f(t)<\sup _{s \in(t-\varepsilon, t)} f(s) .
$$

Alors :

$$
\lambda\left(E_{f}\right)=0 .
$$

Démonstration. - Comme $f$ est continue, tous les points extrémaux de $V_{f}$ appartiennent aussi à $\Gamma_{f}$. D'autre part, si le point vérifie les conditions (3) et (4), alors, grâce au lemme $1,(t, f(t)) \in V_{f}^{o}$ et donc $t$ ne peut pas appartenir à $E_{f}$, ce qui donne (5).

Remarque 1.- Cette proposition, en général, ne s'applique pas aux fonctions dérivables puisque si la condition (A) était vérifiée, on aurait $f^{\prime}(t)=0$ p.p. 
Pour les fonctions lisses on peut proposer les conditions suivantes.

Proposition 2. - Supposons que $f$ est une fonction absolument continue

$$
f(t)=f(0)+\int_{0}^{t} h(s) d s
$$

où $h \in \mathbb{L}^{1}[0,1]$.

Supposons que pour presque tout $t \in[0,1]$ la condition suivante est vérifiée :

(B) Il existe $\varepsilon_{t}>0$ tel que $\forall \varepsilon \in\left(0, \varepsilon_{t}\right)$

$$
\inf _{s \in(t, t+\varepsilon)} \int_{t}^{s}[h(u)-h(t)] d u<0<\sup _{s \in(t, t+\varepsilon)} \int_{t}^{s}[h(u)-h(t)] d u .
$$

Alors on a (5).

Démonstration. - Avant tout on remarque que les conditions (7) sont équivalentes aux inégalités :

$$
\inf _{s \in(t, t+\varepsilon)}\left\{f(s)-f(t)-f^{\prime}(t)(t-s)\right\}<0<\sup _{s \in(t, t+\varepsilon)}\left\{f(s)-f(t)-f^{\prime}(t)(t-s)\right\} .
$$

Supposons que (B) est vérifiée pour un point $t \in(0,1)$. Alors grâce à l'inégalité droite de (8) il existe $s_{1}>t$ tel que $f\left(s_{1}\right)>f(t)+f^{\prime}(t)\left(t-s_{1}\right)$. Grâce à la dérivabilité de $f$ en $t$ il existe $s_{2}<t$ tel que

$$
\frac{f(t)-f\left(s_{2}\right)}{t-s_{2}}<\frac{f\left(s_{1}\right)-f(t)}{s_{1}-t}
$$

Tout cela signifie que le segment joignant les points $\left(s_{2}, f\left(s_{2}\right)\right), \quad\left(s_{1}, f\left(s_{1}\right)\right)$ passe au dessus du point $(t, f(t))$. De la même façon, en utilisant la partie gauche de (8), on trouve deux points $u_{2}<t<u_{1}$ tels que le segment joignant les points $\left(u_{2}, f\left(u_{2}\right)\right), \quad\left(u_{1}, f\left(u_{1}\right)\right)$ passe au dessous du point $(t, f(t))$. Le point $(t, f(t))$ appartient donc à $V_{f}^{o}$ et ne peut pas appartenir à $E_{f}$, ce qui donne (5).

Remarque 2. - On pourrait remplacer les inégalités (7) par les inégalités analogues mais avec $[t, 1]$ remplacé par $[0, t]$.

Proposition 3. - Si f satisfait les hypothèses de la proposition 2 et est dérivable en tout point de $(0,1)$, alors $E_{f}$ est fermé, sans points isolés sur $(0,1)$ et négligeable.

Démonstration. - Puisque $f$ est continue sur $[0,1], \Gamma_{f}$ est compact et $\operatorname{donc} \operatorname{ext}\left(V_{f}\right)$ sera fermé. Il est facile de voir que $x \in E_{f}$ est un point isolé si et seulement si le point extrémal $y$ correspondant est anguleux. Par conséquent, si $f$ est dérivable, l'ensemble $E_{f}$ ne peut pas avoir de points isolés. En effet, dans le cas contraire, il existerait un point extrémal anguleux. Comme ce point devrait appartenir aussi à $\Gamma_{f}$, on arriverait à une contradiction avec la dérivabilité de $f$. 
Remarque 3. - Sous les hypothèses de la proposition 3 l'ensemble $E_{f}$ est un ensemble de mesure de Lebesgue nulle, mais de plus, si l'on exclut le cas trivial quand $E_{f}$ se réduit à $\{0,1\}$, il est cantorien (c.à.d. fermé, nulle part dense et sans points isolés) et donc de puissance du continu.

\subsection{Processus}

Soit $X=\{X(t), t \in[0,1]\}$ un processus mesurable sur $[0,1]$. En utilisant les propositions 1 et 2 et le théorème de Fubini, on obtient les résultats suivants.

COROllaire 1. - Supposons que $X$ est continue et que pour presque chaque $t \in[0,1]$ avec probabilité 1 la condition (A) (avec $f$ remplacé par $X$ ) est vérifiée.

Alors,

$$
\lambda\left(E_{X}\right)=0 \quad \text { p.s. }
$$

COROLlaire 2. - Supposons que $X$ est dérivable et que pour presque tout $t \in[0,1]$ avec probabilité 1 la condition (B) (avec h remplacé par $X^{\prime}$ ) soit vérifiée.

Alors, avec probabilité 1 l'ensemble $E_{X}$ est cantorien et (9) a lieu.

\section{Processus gaussiens}

Dans cette section on applique les conditions suffisantes de la section 2 aux processus gaussiens à accroissements stationnaires. On s'appuie sur un lemme technique sur le supremum des suites gaussiennes.

\subsection{Sur le supremum des suites gaussiennes}

Notons $\rho(\xi, \eta)$ le cofficient de corrélation entre $\xi$ et $\eta$.

LEMME 2. - Soit $\left\{\xi_{n}, n \geqslant 1\right\}$ une suite de v.a. gaussiennes standards, $\xi_{n} \sim \mathcal{N}(0,1)$, vérifiant la condition :

(H1) Il existe $r \in(0,1)$ tel que $\forall m \in \mathbb{N}$

$$
\limsup _{n \rightarrow \infty}\left|\rho\left(\xi_{n}, \xi_{n+m}\right)\right| \leqslant r
$$

Alors

$$
P\left\{\limsup _{n \rightarrow \infty} \xi_{n}=+\infty\right\}=1 .
$$

Démonstration. - Avant tout on remarque que

$$
P\left\{\underset{n \rightarrow \infty}{\limsup } \xi_{n}=+\infty\right\}=1 \quad \text { ou } \quad 0 .
$$

En effet, soit

$$
H=\left\{a \in R^{\mathbb{N}} \mid \exists \eta \in L^{2}(d P), a_{n}=E \eta \xi_{n}, n \in \mathbb{N}\right\} .
$$

Il est bien connu que $H$, appelé le noyau de $\left(\xi_{n}\right)$, représente l'ensemble des translations admissibles pour la suite $\left(\xi_{n}\right)$. Comme tous les éléments de $H$ sont des suites bornées, 
l'ensemble $\left\{x \in R^{\mathbb{N}} \mid \limsup _{n \rightarrow \infty} x_{n}=+\infty\right\}$ sera invariant par rapport à $H$, ce qui donne (12) grâce au théorème 5.9 [9].

Soit $r_{1}$ un nombre de l'intervalle $(r, 1)$. Grâce à $(\mathrm{H} 1)$, pour chaque $m \in \mathbb{N}$, il existe $n_{0} \in \mathbb{N}$ tel que pour tout $n \geqslant n_{0}$ et tout $i, j=1, \ldots, m$,

$$
\left|\rho\left(\xi_{n+i}, \xi_{n+j}\right)\right|<r_{1} .
$$

Ceci signifie que l'ensemble $\left\{n_{0}+i, 1 \leqslant i \leqslant m\right\}$ est $\varepsilon$-distinguable avec $\varepsilon=\sqrt{2-2 r_{1}}$, pour la distance $d_{\xi}$ associée à la suite $\left(\xi_{n}\right)$ sur $\mathbb{N}$. On en déduit en termes d'entropie

$$
N\left(\varepsilon / 2, \mathbb{N}, d_{\xi}\right)=\infty
$$

En appliquant le théorème 6.14 [9], on en déduit que $P\left\{\limsup _{n \rightarrow \infty} \xi_{n}=+\infty\right\} \geqslant 1 / 2$. Compte tenu la remarque précédente, cela donne (11).

Du lemme 2 on déduit directement

LEMME 3. - Soit $\left\{\xi_{n}, n \geqslant 1\right\}$ une suite gaussienne centrée vérifiant la condition (H1) ci-dessus. Alors

$$
P\left\{\sup _{n} \xi_{n}>0\right\}=1 .
$$

Démonstration. - En effet, grâce au lemme 2

$$
\limsup _{n} \frac{\xi_{n}}{\sqrt{\operatorname{Var} \xi_{n}}}=+\infty \quad \text { p.s. }
$$

Soit $B=\left\{\sup _{n} \xi_{n} \leqslant 0\right\}$. Il est clair que sur l'ensemble $B$ on a l'inégalité $\sup _{n} \frac{\xi_{n}}{\sqrt{\operatorname{Var} \xi_{n}}} \leqslant 0$. Donc, $\mathbb{P}(B)=0$, ce qui est équivalent à (14).

Le lemme suivant est une adaptation du lemme 1 aux processus.

Lemme 4. - Soit $X=\{X(t), t \geqslant 0\}$ un processus gaussien centré séparable tel que $X(0)=0$. Supposons que la condition suivante est vérifiée :

(H2) Il existe $r \in(0,1)$ et une suite $\left\{t_{k}\right\}, t_{k} \downarrow 0$, tels que $\forall k$

$$
\limsup _{s \rightarrow 0}\left|\rho\left(X\left(t_{k}\right), X(s)\right)\right| \leqslant r .
$$

Alors

$$
\limsup _{s \rightarrow 0} \frac{X(s)}{\sqrt{\operatorname{Var} X(s)}}=+\infty, \quad \text { p.s. }
$$

et pour chaque $\varepsilon>0$

$$
\sup _{t \in[0, \varepsilon]} X(t)>0 \quad \text { p.s. }
$$

Démonstration. - En effet, il est facile de voir que la condition (H2) permet de choisir une sous-suite $\left(k_{n}\right) \subset \mathbb{N}$ telle que pour la suite $\left\{X\left(t_{k_{n}}\right)\right\}$ la condition (H1) soit vérifiée. Alors par le lemme 2 on obtient (16) et par le lemme 3 pour chaque $m$ on a $\sup _{n \geqslant m} X\left(t_{k_{n}}\right)>0$ p.s., ce qui prouve le lemme 4 . 
Remarque 4. - Il y a beaucoup de travaux consacrés à l'étude du supremum de processus gaussiens (voir, par exemple, [4] et ses références). Il est connu, en particulier, ([3], Th.7.3), que pour un processus gaussien continu $\{X(t), 0 \leqslant t \leqslant T\}$ issu de zéro $(X(0)=0)$ et non dégénéré pour $t>0$, c'est-à-dire $E X^{2}(t)>0, t>0$, la loi de la v.a. $M=\sup _{0 \leqslant t \leqslant T} X(t)$ est concentrée sur $[0, \infty)$ et est absolument continue sur $(0, \infty)$. Il existe des exemples montrant que cette loi peut avoir un atome au point 0 . Dans ce contexte on peut considérer le lemme 4 comme un résultat proposant des conditions suffisantes pour la continuité absolue de la loi de $M$.

\subsection{Conditions du type spectral}

Dans cette section on considère des processus gaussiens centrés $X=\left\{X(t), t \in \mathbb{R}^{1}\right\}$, dont la fonction de corrélation admet la représentation suivante

$$
K(t, s)=\int_{\mathbb{R}^{1}} g(s, x) \overline{g(t, x)} F(d x),
$$

où $F$ est une mesure $\sigma$-finie sur $\mathbb{R}^{1}$ et pour tout $s$ la fonction $x \rightarrow g(s, x)$ appartient à $\mathbb{L}^{2}(d F)$.

Suivant la terminologie de [9], cela signifie que le système de fonctions $\{g(s,),. s \in$ $\mathbb{R}^{1}$ \} est un $\mathbb{L}^{2}(d F)$-modèle du processus $X$.

On va proposer des conditions pour que

$$
\forall \varepsilon>0, \quad P\left\{\sup _{t \in[0, \varepsilon]}(X(t)-X(0))>0\right\}=1 .
$$

THÉORÈME 1. - Soit $X$ un processus gaussien centré dont la fonction de corrélation admet la représentation (17). Supposons que la mesure $F$ et la fonction $G(t, x)=$ $g(t, x)-g(0, x)$ satisfont les conditions :

1) Pour tout $s$

$$
M(s) \stackrel{\text { déf }}{=} \sup _{x}|G(s, x)|<+\infty .
$$

2) Il existe des constantes $A, C_{1}, C_{2}, t_{0}>0$ et deux fonctions $\varphi$ et $\psi, \mathbb{R}_{+} \rightarrow \mathbb{R}_{+}$, telles que :

$$
F\left(\left\{x|| x \mid \geqslant t_{0}\right\}\right)<\infty
$$

$\psi$ est croissante, $\lim _{x \rightarrow+\infty} \psi(x)=+\infty$;

$$
\begin{gathered}
\int_{|x| \leqslant t_{0}} \psi^{2}(|x|) F(d x)<+\infty ; \\
\int_{|x| \geqslant t_{0}} \psi^{2}(|x|) F(d x)=+\infty ;
\end{gathered}
$$

pour tous $t, x$ tels que $|x t| \in[0, A]$,

$$
C_{1} \varphi(t) \psi(x) \leqslant|G(t, x)| \leqslant C_{2} \varphi(t) \psi(x) .
$$


Alors on a (18).

Les conditions de ce théorème se vérifient facilement dans les cas concrets. On donnera quelques exemples ci-dessous.

Démonstration. - Fixons $s$ et montrons que

$$
\delta(t) \stackrel{\text { déf }}{=} \rho(X(t)-X(0), X(s)) \underset{t \rightarrow 0}{\longrightarrow} 0 .
$$

Posons $D^{2}(t)=\int_{\mathbb{R}}|G(t, x)|^{2} F(d x), \quad T=A / t, A$ étant la constante de la condition 2) du théorème. En utilisant la représentation (17), on trouve

$$
\delta(t)=\frac{\int_{\mathbb{R}} G(t, x) \overline{G(s, x)} F(d x)}{D(t) D(s)}=\delta_{1}(t)+\delta_{2}(t)+\delta_{3}(t),
$$

où

$$
\begin{aligned}
\delta_{1}(t) & =\frac{\int_{|x| \leqslant t_{0}} G(t, x) G(s, x) F(d x)}{D(t) D(s)}, \quad \delta_{2}(t)=\frac{\int_{t_{0} \leqslant|x| \leqslant T} G(t, x) G(s, x) F(d x)}{D(t) D(s)}, \\
\delta_{3}(t) & =\frac{\int_{T<|x|} G(t, x) G(s, x) F(d x)}{D(t) D(s)} .
\end{aligned}
$$

Estimation de $\delta_{1}(t)$. On déduit directement de la condition 2) et Cauchy-Schwartz pour $t$ assez petit :

$$
\left|\delta_{1}(t)\right|^{2} \leqslant \frac{2 t_{0} M^{2}(s) \int_{|x| \leqslant t_{0}}|G(t, x)|^{2} F(d x)}{D^{2}(s) \int_{t_{0}<|x| \leqslant T}|G(t, x)|^{2} F(d x)} \leqslant C \frac{\int_{|x| \leqslant t_{0}} \psi^{2}(x) F(d x)}{\int_{t_{0}<|x| \leqslant T} \psi^{2}(x) F(d x)},
$$

ce qui converge vers 0 quand $t \rightarrow 0$.

Estimation de $\delta_{2}(t)$. On trouve en utilisant (19)

$$
\left|\delta_{2}(t)\right|^{2} \leqslant C \frac{M^{2}(s)}{D^{2}(s)} \frac{\left[\int_{t_{0} \leqslant|x| \leqslant T}|G(t, x)| F(d x)\right]^{2}}{\int_{\mathbb{R}_{+}}|G(t, x)|^{2} F(d x)} .
$$

Sans perdre de généralité on peut supposer que la mesure $F$ est concentrée sur $\mathbb{R}_{+}$et $G(t, x)$ est positive. Sinon on pourrait remplacer $F$ par la mesure $F_{1}(B)=$ $F(B)+F(-B), B \in \mathcal{B}_{\mathbb{R}_{+}}$et $|G(t, x)|$ par $G_{1}(x)=|G(t, x)|+|G(t,-x)|, x \in \mathbb{R}_{+}$.

On a maintenant

$$
\begin{aligned}
{\left[\int_{t_{0}}^{T} G(t, x) F(d x)\right]^{2} } & =2 \int_{t_{0} \leqslant y \leqslant x \leqslant T} G(t, x) G(t, y) F(d x) F(d y) \\
& =2 \int_{t_{0}}^{T} G(t, x) \tilde{G}(t, x) F(d x),
\end{aligned}
$$


où

$$
\tilde{G}(t, x)=\int_{t_{0}}^{x} G(t, y) F(d y)
$$

Grâce à la condition 2)

$$
\frac{\int_{t_{0}}^{T} G(t, x) \tilde{G}(t, x) F(d x)}{\int_{t_{0}}^{T} G^{2}(t, x) F(d x)} \leqslant C \frac{\int_{t_{0}}^{T} \psi(x)\left[\int_{t_{0}}^{x} \psi(y) F(d y)\right] F(d x)}{\int_{t_{0}}^{T} \psi^{2}(x) F(d x)} .
$$

Comme $\forall t_{1}>t_{0}$

$$
\limsup _{x \rightarrow \infty} \frac{\int_{t_{0}}^{x} \psi(y) F(d y)}{\psi(x)} \leqslant F\left(\left[t_{1}, \infty\right]\right)
$$

on trouve que

$$
\int_{t_{0}}^{x} \psi(y) F(d y)=\mathrm{o}(\psi(x)), \quad x \rightarrow+\infty .
$$

Cette relation avec (26) et (27) donne la convergence $\delta_{2}(t) \rightarrow 0$ quand $t \rightarrow 0$.

Estimation de $\delta_{3}(t)$. Elle découle directement de (20) et de l'inégalité CauchySchwartz :

$$
\begin{aligned}
{\left[\int_{T}^{\infty} G(t, x) F(d x)\right]^{2} } & \leqslant \int_{T}^{\infty} F(d x) \cdot \int_{\mathbb{R}^{1}} G^{2}(t, x) F(d x) \\
& =\mathrm{o}\left(\int_{\mathbb{R}^{1}} G^{2}(t, x) F(d x)\right), \quad T \rightarrow \infty,
\end{aligned}
$$

d'où $\delta_{3}(t) \rightarrow 0, t \rightarrow 0$.

On a donc $\delta(t) \rightarrow 0, t \rightarrow 0$. En appliquant le lemme 4, on obtient (18).

\subsection{Processus à accroissements stationnaires}

Soit $X=\left\{X(t), t \in \mathbb{R}_{+}\right\}$un processus gaussien centré à accroissements stationnaires (à.a.s.). Il est bien connu (voir, par exemple, [7], V, §4, Th. 3 et I, §5, Th. 4) qu'il admet la représentation spectrale

$$
X(t)=\int_{\mathbb{R}^{1}} \frac{\mathrm{e}^{\mathrm{i} t x}-1}{\mathrm{i} x} \sqrt{1+x^{2}} Z(d x),
$$

où $Z$ est une mesure stochastique orthogonale telle que :

$$
E Z^{2}((a, b])=\tilde{F}((a, b])
$$

$\tilde{F}$ étant une mesure finie symétrique sur $\mathbb{R}^{1}$. 
ThÉORÈme 2. - Soit X un processus gaussien centré à.a.s. Supposons qu'il est continu mais non dérivable. Alors

$$
\lambda\left(E_{X}\right)=0 \quad \text { p.s. }
$$

Remarquons que la non dérivabilité de $X$ est équivalente à

$$
\int_{\mathbb{R}} x^{2} \tilde{F}(d x)=+\infty .
$$

Démonstration. - On déduit de (28) la représentation suivante pour la fonction de corrélation de $X$ :

$$
R(t, s)=\int_{\mathbb{R}^{1}} g(t, x) \overline{g(s, x)} F(d x),
$$

où

$$
g(t, x)=\mathrm{e}^{\mathrm{i} t x}-1, \quad F(d x)=\frac{1+x^{2}}{x^{2}} \tilde{F}(d x) .
$$

On va vérifier les conditions du Théorème 1 . La mesure $F$, évidemment, est $\sigma$-finie et $\forall t_{0}>0, F\left(\left[t_{0}, \infty\right)\right)<+\infty$. On a

$$
\forall t, x, \quad G(t, x)=\left|\mathrm{e}^{\mathrm{i} t x}-1\right| \leqslant 2
$$

ce qui donne (19).

Si l'on prend $A=\pi, \varphi(t)=t, \psi(x)=x$, alors (23) aura lieu avec $C_{1}=2 / \pi, C_{2}=1$. La condition (22) est vérifiée grâce à (30).

Le théorème 1 s'applique donc et on a (18). Par stationnarité des accroissements et normalité, pour presque chaque $t \in[0,1]$ avec probabilité 1 la condition (A) est vérifiée et par le corollaire 1 on arrive à (29).

\subsection{Processus intégrés}

Considérons maintenant les processus du type

$$
Y(t)=\int_{0}^{t} X(s) d s,
$$

où $X$ est un processus gaussien.

THÉORÈme 3. - Supposons que $X$ est un processus gaussien centré à accroissements stationnaires dont les trajectoires sont continues mais pas dérivables. Alors presque sûrement $E_{Y}$ est un ensemble cantorien et $\lambda\left(E_{Y}\right)=0$.

Démonstration. - On va montrer d'abord que pour tout $\varepsilon>0$ avec probabilité 1

$$
\sup _{s \in[0, \varepsilon]}\left\{Y_{s}\right\}>0 .
$$


On déduit de (28) que la fonction de corrélation $R_{Y}$ du processus $Y$ admet la représentation

$$
R_{Y}(t, s)=\int_{\mathbb{R}^{1}}\left(\mathrm{e}^{\mathrm{i} t x}-1-\mathrm{i} t x\right)\left(\overline{\mathrm{e}^{\mathrm{i} s x}-1-\mathrm{i} s x}\right) \frac{1+x^{2}}{x^{4}} \tilde{F}(d x),
$$

où $\tilde{F}$ est une mesure finie symétrique sur $\mathbb{R}^{1}$ telle que $\int_{\mathbb{R}} x^{2} \tilde{F}(d x)=+\infty$.

Posons

$$
\begin{aligned}
& g(t, x)=\frac{\mathrm{e}^{\mathrm{i} t x}-1-\mathrm{i} t x}{x}, \quad g(0, x)=0, \quad G(t, x)=\left|\frac{\mathrm{e}^{\mathrm{i} t x}-1-\mathrm{i} t x}{x}\right|, \\
& F(d x)=\frac{1+x^{2}}{x^{2}} \tilde{F}(d x) .
\end{aligned}
$$

Alors les conditions du théorème 1 seront remplies, si l'on prend $A=\pi, C_{1}=2 / \pi^{2}$, $C_{2}=1 / 2, \varphi(t)=t^{2}, \psi(x)=x, t_{0}=1$. On a donc (33).

Pour démontrer notre théorème il nous suffit, grâce au corollaire 2 , de vérifier la condition (B). Si l'on pose $\tilde{X}(u)=X(t+u)-X(t)$, la partie droite de cette condition peut être récrite comme

$$
P\left\{\sup _{s \in[0, \varepsilon]} \int_{0}^{s} \tilde{X}(u) d u>0\right\}=1 .
$$

Pour $t$ fixé les accroissements du processus $\tilde{X}$ sont stationnaires, ses trajectoires sont continues et non dérivables. Cela signifie qu'il satisfait les conditions du théorème. En appliquant la première partie de la démonstration, on obtient (35), d'où le résultat.

Remarque 5. - Puisque chaque processus stationnaire a aussi des accroissements stationnaires, les théorèmes 2 et 3 s'appliquent également aux processus stationnaires.

Remarque 6. - Le processus $W_{H}$ du mouvement brownien fractionnaire est continu, non dérivable et à accroissements stationnaires. Par conséquent, le théorème 2 s'applique à $W_{H}$ et le théorème 3 s'applique à $Y_{H}(t)=\int_{0}^{t} W_{H}(s) d s$.

\section{Enveloppes convexes des processus transformés}

Dans cette section on va montrer que les propriétés en discussion des enveloppes convexes sont, dans un certain sens, stables par transformations lisses.

Proposition 4. - Soit $f:[0,1] \rightarrow \mathbb{R}^{1}$ une fonction continue telle que la propriété (A) soit vérifiée dans $t_{0} \in(0,1)$. Soit $\varphi: \mathbb{R}^{1} \rightarrow \mathbb{R}^{1}$ une fonction pour laquelle $\varphi^{\prime}\left(f\left(t_{0}\right)\right)$ existe et $\varphi^{\prime}\left(f\left(t_{0}\right)\right) \neq 0$.

Alors la propriété (A) sera aussi vérifiée au point t $t_{0}$ pour la composée $F=\varphi \circ f$.

Démonstration. - Par hypothèse

$$
F\left(t_{0}+h\right)-F\left(t_{0}\right)=\varphi^{\prime}\left(f\left(t_{0}\right)\right)\left(f\left(t_{0}+h\right)-f\left(t_{0}\right)\right)+R(h),
$$


où $R(h)=\mathrm{o}\left(f\left(t_{0}+h\right)-f\left(t_{0}\right)\right), h \rightarrow 0$.

Puisque $\varphi^{\prime}\left(f\left(t_{0}\right)\right) \neq 0$, il existe $\varepsilon>0$ tel que pour $|h|<\varepsilon$

$$
|R(h)| \leqslant \frac{\left|\varphi^{\prime}\left(f\left(t_{0}\right)\right)\right|}{2}\left|f\left(t_{0}+h\right)-f\left(t_{0}\right)\right| .
$$

Sans perdre de généralité on peut supposer que $\varphi^{\prime}\left(f\left(t_{0}\right)\right)>0$. Maintenant si $h$ est tel que $f\left(t_{0}+h\right)>f\left(t_{0}\right)$, on a par (36)

$$
F\left(t_{0}+h\right)-F\left(t_{0}\right)>\frac{\varphi^{\prime}\left(f\left(t_{0}\right)\right)}{2}\left(f\left(t_{0}+h\right)-f\left(t_{0}\right)>0 .\right.
$$

Le cas $f\left(t_{0}+h\right)<f\left(t_{0}\right)$ se traite de la même façon.

Corollaire 3. - Soit $X$ un processus gaussien centré à.a.s. Supposons qu'il est continu mais non dérivable. Soit $\varphi: \mathbb{R}^{1} \rightarrow \mathbb{R}^{1}$ une fonction telle que $\varphi^{\prime}(x)$ existe et $\varphi^{\prime}(x) \neq 0$ pour presque tout $x \in \mathbb{R}^{1}$. Alors pour le processus $Y(t)=\varphi(X(t))$ on a

$$
\lambda\left(E_{Y}\right)=0 \quad \text { p.s. }
$$

Démonstration. - De la représentation (28) on déduit facilement que l'ensemble $K_{X}$ de points $t$ pour lesquels $\operatorname{Var} X(t)=0$ est au plus dénombrable. Posons $K=\left\{y \mid \varphi^{\prime}(y)=\right.$ $0\}$. Puisque $\lambda(K)=0, \varphi^{\prime}(X(t)) \neq 0$ p.s. pour tous $t \in K_{X}^{c}$, donc $\varphi^{\prime}(X(t)) \neq 0$ p.s. pour presque tous $t \in[0,1]$. Dans la démonstration du théorème 2 on a vu que pour presque chaque $t \in[0,1]$ avec probabilité 1 la condition (A) pour le processus $X$ est vérifiée. La proposition 4 nous montre alors que pour $Y$ la propriété (A) sera vérifiée p.s. pour presque tout $t \in[0,1]$, et par le corollaire 1 on obtient (37).

On passe aux processus lisses.

Proposition 5. - Soit Y un processus gaussien centré dont les trajectoires sont absolument continues :

$$
Y(t)=Y(0)+\int_{0}^{t} X(s) d s .
$$

Supposons que $\operatorname{Var} X(t)>0$ pour presque tout $t \in[0,1]$ et que la condition (8) (avec $f$ remplacé par $Y$ ) soit vérifiée p.s. Supposons que pour presque tout $t$

$$
\limsup _{h \rightarrow 0} \frac{\Delta(h)}{h^{2}}=+\infty \quad \text { p.s. }
$$

où $\Delta(h)=Y(t+h)-Y(t)-X(t) h$.

Soit $\varphi: \mathbb{R}^{1} \rightarrow \mathbb{R}^{1}$ est une fonction deux fois dérivable et telle que $\varphi^{\prime}(y) \neq 0$ p.p.

Alors pour le processus $Z(t)=\varphi(Y(t))$ l'ensemble $E_{Z}$ est p.s. cantorien et $\lambda\left(E_{Z}\right)=0$.

Remarque 7. - La condition (38) entraîne la non dérivabilité du processus $X$. 
Remarque 8. - Par la normalité du processus $\Delta$ on déduit de (38) que pour presque tout $t$

$$
\liminf _{h \rightarrow 0} \frac{\Delta(h)}{h^{2}}=-\infty \text { p.s. }
$$

Preuve de la proposition 5. - Il est facile de voir que si la dérivée d'une fonction absolument continue est non-nulle $p . p$., alors cette fonction elle-même est non nulle $p . p .$. La condition $« X(t)>0$ pour presque tout $t \in[0,1] »$ entraîne donc la même condition pour $Y$.

Soit maintenant $t_{0} \in(0,1)$ et $\omega_{0} \in \Omega$ des points tels que : $Y^{\prime}\left(t_{0}\right)$ existe, $Y^{\prime}\left(t_{0}\right)$ et $Y\left(t_{0}\right)$ sont non-dégénérées, les conditions (8) (pour $Y$ ) et (38) sont vérifiées en $t_{0}$. Grâce à ce choix $\varphi^{\prime}\left(Y\left(t_{0}\right)\right) \neq 0$ p.s. On va montrer que (8) aura lieu en $t_{0}$ et $\omega_{0}$ pour le processus $Z$.

Comme $\varphi$ est deux fois dérivable,

$$
\psi(h) \stackrel{\text { déf }}{=} Z\left(t_{0}+h\right)-Z\left(t_{0}\right)-Z^{\prime}\left(t_{0}\right) h=\varphi^{\prime}\left(Y\left(t_{0}\right)\right) \Delta(h)+R(h),
$$

où

$$
\begin{aligned}
R(h) & =\varphi^{\prime \prime}\left(Y\left(t_{0}\right)\right) \frac{\left[Y\left(t_{0}+h\right)-Y\left(t_{0}\right)\right]^{2}}{2}(1+\mathrm{o}(1)) \\
& =\varphi^{\prime \prime}\left(Y\left(t_{0}\right)\right) \frac{Y^{\prime}\left(t_{0}\right)^{2}}{2} h^{2}(1+\mathrm{o}(1))=\mathrm{O}\left(h^{2}\right), \quad h \rightarrow 0 .
\end{aligned}
$$

Supposons que $\varphi^{\prime}\left(Y\left(t_{0}\right)\right)>0$. Étudions le comportement de $\psi(h)$ pour $h \downarrow 0$. Si pour un $h_{0}>0$ on avait pour tous $h \in\left(0, h_{0}\right)$ l'inégalité $\psi(h) \leqslant 0$, on aurait grâce à (40) pour un $K>0$

$$
\Delta(h) \leqslant K h^{2}, \quad h \in\left(0, h_{0}\right),
$$

en contradiction avec (38).

La démonstration de la deuxième partie de (8) et le cas $\varphi^{\prime}\left(Y\left(t_{0}\right)\right)<0$ se traitent de la même façon : il suffit d'utiliser la remarque 8 .

COROLlaire 4. - Soit $Y$ un processus gaussien centré à.a.s. Supposons qu'il est absolument continu et tel que $Y^{\prime}$ est continu mais non dérivable. Supposons que $\varphi: \mathbb{R}^{1} \rightarrow$ $\mathbb{R}^{1}$ est une fonction deux fois dérivable et telle que $\varphi^{\prime}(y) \neq 0$ p.p.

Alors pour le processus $Z(t)=\varphi(Y(t))$ l'ensemble $E_{Z}$ est p.s. cantorien et $\lambda\left(E_{Z}\right)=0$.

Démonstration. - En appliquant le théorème 1 et le lemme 4, on obtient facilement le lemme suivant.

Lemme 5. - Supposons que le processus Z vérifie les conditions du corollaire 4. Alors

$$
\limsup _{h \rightarrow 0} \frac{Z(h)-Z(0)}{h^{2}}=+\infty \quad \text { p.s. }
$$


Maintenant, comme

$$
Y(t+h)-Y(t)-Y^{\prime}(t) h=\int_{t}^{t+h}\left(Y^{\prime}(s)-Y^{\prime}(t)\right) d s,
$$

on obtient (38) en appliquant le lemme 5 au processus

$$
Z(h)=\int_{t}^{t+h}\left(Y^{\prime}(s)-Y^{\prime}(t)\right) d s .
$$

\section{Conclusion}

1. Les exemples des processus gaussiens considérés ci-dessus montrent que les propriétés de l'ensemble $E_{X}$ dépendent d'abord des propriétés locales des trajectoires de $X$. La règle générale est la suivante : plus les trajectoires sont lisses, plus massif sera l'ensemble $E_{X}$. En effet, la situation typique pour des processus non dérivables est telle que $\lambda\left(E_{X}\right)=0$ p.s., même il semble probable que souvent cet ensemble est au plus dénombrable. Si les trajectoires sont dérivables une seule fois, $E_{X}$ est cantorien (et donc de puissance du continu!), mais de mesure de Lebesgue zéro. Il est facile de comprendre que si la dérivée seconde existe et est continue, alors l'ensemble $E_{X}$ contient des segments.

2. Cette tendance se confirme également par l'étude d'autres classes de processus (processus gaussiens transformés - voir section 4, processus à accroissements indépendants, martingales locales, champs gaussiens, ...). La plupart des résultats concernant ces classes ne figure pas dans cet article par cause de limitation de volume. Ils seront publiés ailleurs.

3. Dans tous les résultats présentés, on a supposé que la moyenne du processus initial $X$ est égale à 0 . On a fait cette hypothèse par commodité et parce que le passage de $X$ à $X+a$, où $a(t)$ est une fonction déterministe, n'est d'habitude pas difficile.

Par exemple, si $a$ définit une translation admissible pour la loi de $X$ (c.à.d. si la loi de $X+a$ est équivalente à la loi de $X$ ), les propriétés p.s. des ensembles $E_{X}$ et $E_{X+a}$ seront les mêmes. Souvent on peut même en dire plus. Supposons que pour presque tout $t \in[0,1]$ avec probabilité 1

$$
\begin{aligned}
& \limsup _{h \downarrow 0} \frac{X(t+h)-X(t)}{h}=+\infty, \\
& \liminf _{h \downarrow 0} \frac{X(t+h)-X(t)}{h}=-\infty
\end{aligned}
$$

avec relations analogues à gauche de $t$.

Alors, si $a$ est une fonction dérivable $p . p$., on a les mêmes relations pour $X+a$. Comme ces relations entraînent (3), par le corollaire $1 \lambda\left(E_{X+a}\right)=0$.

4. Il reste encore beaucoup de questions ouvertes sur la structure de l'ensemble $E_{X}$. En voici deux : 
i) Il serait intéressant de trouver des conditions suffisantes qui garantissent l'absence de points extrémaux isolés.

ii) Quelle est la dimension de Hausdorff de $E_{X}$ ?

\section{Remerciements}

Je tiens à remercier A. Dermoune, Ch. Suquet pour l'intérêt qu'ils ont porté à ce travail, ainsi que M. Lifshits et le rapporteur anonyme pour les remarques utiles qui ont permis d'abréger la preuve initiale du lemme 2 et d'améliorer le texte de l'article.

\section{RÉFÉRENCES}

[1] M. Avellanede, E. Weinan, Statistical properties of shocks in Burgers turbulence, Comm. Math. Phys. 172 (1995) 13-38.

[2] J. Bertoin, Structure of shocks in Burgers turbulence with stable noise initial data, Comm. Math. Phys. 203 (1999) 729-741.

[3] Yu. Davydov, M. Lifshits, Stratification method in some probability problems, J. Soviet. Math. 37 (1) (1987).

[4] Yu. Davydov, M. Lifshits, N. Smorodina, Local Properties of Distributions of Stochastic Functionals, American Mathematical Society, 1998.

[5] Yu. Davydov, V. Paulauskas, A. Račkauskas, More on $p$-stable convex random sets in Banach spaces, J. Theoret. Probab. 13 (1) (2000) 39-64.

[6] Yu. Davydov, A. Vershik, Réarrangements convexes des marches aléatoires, Ann. I. H. P. 34 (1) (1998) 73-95.

[7] I. Gikhman, A. Skorohod, Introduction to the Theory of Random Processes, Saunders, Philadelphia, 1969.

[8] P. Groenboom, The concave majorant of Brownian motion, Ann. Probab. 11 (4) (1983) 1016-1027.

[9] M.A. Lifshits, Gaussian Random Functions, Kluwer, Dordrecht, 1995.

[10] J.W. Pitman, Remarks on the convex minorant of Brownian Motion, in: E. Cinlar, K.L. Chung, R.K. Getoor (Eds.), Seminar on Stochastic Processes, Birkhauser, Basel, 1982, pp. 219-227.

[11] R. Ryan, The statistics of Burgers turbulence initialized with fractional Brownian noise data, Comm. Math. Phys. 191 (1998) 71-86.

[12] R. Ryan, M. Avellanede, The one-point statistics of viscous Burgers turbulence initialized with Gaussian data, Comm. Math. Phys. 200 (1999) 1-23.

[13] Ya. Sinai, Statistics of shocks in solutions of inviscid Burgers equation, Comm. Math. Phys. 148 (1992) 6-621.

[14] D. Slepian, The one-sided barier problem for Gaussian noise, Bell. Syst. Tech. J. 41 (2) (1962) 463-501. 